\title{
Ciudadanía mediática en tiempos de crisis. Análisis de la participación sociopolítica de nativos e inmigrantes digitales en la era Post-Web ${ }^{1}$
}

\author{
Grijalva-Verdugo, Abel Antonio²
}

Recibido: 15 de septiembre de 2017 / Aceptado: 8 de noviembre de 2017

Resumen. En la era Post-Web, la participación de las audiencias en asuntos sociopolíticos ha estado marcada por la intermitencia. Esta investigación indaga las posibles relaciones de la ciudadanía mediática ante el constructo teórico participación sociopolítica. Se presentan los hallazgos de un estudio cuantitativo realizado en una entidad (provincia) mexicana, utilizando pruebas no paramétricas ( $U$ de Mann-Whitney, Chi-cuadrado y Kruskal Wallis); el objetivo es identificar si el manejo de medios digitales incide en la percepción y acción comunicativa para el cambio de las audiencias contemporáneas (nativos e inmigrantes digitales). Los resultados sugieren que el binomio participación-tecnología es más complejo de lo que aparenta.

Palabras clave: Ciudadanía mediática; pantallas; participación sociopolítica; nativos digitales; comunicación política.

\section{[en] Media citizenship in times of crisis. Analysis of the sociopolitical participation of Mexicans in the Post-Web Era}

\begin{abstract}
In the Post-web era, the audience's participation in socio-political issues has been intermittent. This work inquires the possible relationships between Media citizenship and its digital culture in face of the theoretical construct of socio-political participation. Present here are the findings of a quantitative study made in a Mexican state, using non-parametric tests (Mann-Whitney U test, Chi-squared and Kruskal Wallis); the objective is to identify if digital media influences communicative perception and action for the change of the contemporary audiences (digital natives and immigrants). The results suggest that the binomial participation-technology is more complex than it seems.
\end{abstract}

Keywords: Media citizenship; screens; sociopolitical participation; digital natives; political communication.

Sumario. 1. Introducción; coordenadas de la investigación. 2. La era Post-Web; la paradoja comunicativa. 3. ¿Nativos \& inmigrantes digitales? 4. Método e instrumentos. 5. Resultados y discusión. 6. Conclusiones. 7. Referencias bibliográficas. 8. Agradecimientos.

\footnotetext{
1 Proyecto financiado por el Programa para el Desarrollo Profesional Docente para el Tipo Superior 2016 (PRODEP), folio UDO-EXB-196. El artículo fue producido durante la estancia posdoctoral del autor con apoyo de la beca "Merit" del Gobierno de Canadá, a través del Fondo de Investigación del Ministerio de la Enseñanza Superior de Quebec (Ministère de l'Éducation et de l'Enseignement supérieur du Québec) durante 2017.

${ }^{2}$ Universidad de Occidente (México).

abel.grijalba@udo.mx,
} 
Cómo citar: Grijalva-Verdugo, Abel Antonio (2017). "Ciudadanía mediática en tiempos de crisis. Análisis de la participación sociopolítica de nativos e inmigrantes digitales en la era Post-Web", en Mediaciones Sociales, 16, 245-262.

\section{Introducción; coordenadas de la investigación}

Para Foucault (2005), la caracterización del poder implica un desempoderamiento de la distribución de las fuerzas de dominio de un sistema, se constituye por la proliferación de imperceptibles impulsos que mantienen la macroestructura a salvo. Sin embargo, el poder no se liga, necesariamente, a fuerzas centrales de cohesión sistémica mediante eslabones económicos, en ocasiones dichas fuerzas se transforman en significaciones sutiles que brindan a la sociedad placeres para identificarles con la estructura (llámense paliativos del capitalismo) para que, eventualmente, sean ellos quienes perpetúan su vigencia.

En ese sentido, la modernidad ha traído novedosas formas de expandir el poder, incluso ideas evocadoras de conciencia individual y colectiva por concepto de la conectividad tecnológica, sobre todo al tratarse de plataformas informativas y comunicativas asentadas en Internet. Con ello, el término transparencia se ha convertido en lugar común del discurso social y es que, el uso de la tecnología favorece el cambio de paradigma de acciones comunicativas tradicionalmente dominadas por emisores privilegiados, hoy los ciudadanos mediáticos caracterizan sus propios modos de vivir en comunidad y exigirse en ella, pues el uso tecnológico facilita las vías para acercarse a las decisiones de lo público, es decir, el juego de la comunicación mediada expandida a todos los sectores.

Precisamente, esta investigación discute la expresión cultura digital en la nueva ciudadanía; y es que la utilización de medios para la vida cotidiana, la educación, el gobierno y el entretenimiento, es innegable. El fenómeno se contextualiza desde la era Post-Web; locución representada, evidentemente, por el elevado consumo de Internet y las TIC (pantallas digitales como teléfonos celulares, tabletas, televisores de realidad aumentada, videojuegos, computadoras, etc.) asimismo, por la crisis producida por el exceso informativo, la hipercomunicación y la hipermodernidad (Lipovetsky \& Serroy, 2009). Fenómenos que, frecuentemente, son ligados a causas sociopolíticas como la baja participación ciudadana en la toma de decisiones y la desconfianza social hacia las instituciones del Estado, incluidos los medios de comunicación (tradicionales como no tradicionales), por lo que a la utopía de la transparencia habría que anteponer la exigencia de consumo y reproducción de fuerzas dominantes de los sistemas simbólico, económico y cultural de la posmodernidad.

A partir de este marco referencial, se exponen los hallazgos de una investigación empírica efectuada en el estado de Sinaloa (México) donde prevalecen condiciones de participación social baja. Tal como afirman las investigaciones de Grijalva-Verdugo \& Moreno-Candil (2017), Ruelas (2016) y Grijalva-Verdugo \& Mondaca-Cota (2017) y que, aluden a referenciales de violencia física y simbólica emanadas del narcotráfico de la región.

El instrumento utilizado es un cuestionario tipo escala aplicado en el 2016; capta los constructos teóricos cultura digital y participación sociopolítica con el 
objetivo de identificar si la premisa o "ideal" asociado a la tecnología en la transparencia, participación y pensamiento crítico de la ciudadanía mediática es, necesariamente, relevante o en qué medida lo es. Los resultados se organizan desde tres preguntas guía: P1) ¿existen relaciones entre la escolaridad de los sujetos y los usos de medios digitales? P2) ¿de qué manera la cultura digital influye en la acción social de los nativos e inmigrantes digitales? y P3) ¿hay diferencias estadísticas desde las variables de agrupación uso de medios digitales y grupo etario con las formas de percibir la participación en redes sociales?

Los objetivos del artículo residen en debatir las intersecciones entre mediación tecnológica y la participación sociopolítica de las audiencias, la finalidad es ofrecer datos empíricos obtenidos en una localidad mexicana y que, pueden ser referenciados, sobre todo, en otras poblaciones de América Latina o el mundo.

\section{La era Post-Web; la paradoja comunicativa}

Desde la invención de la fotografía, incluso desde la aparición de las primeras pinturas rupestres, el ser humano ha evidenciado su elocuente fascinación por capturar la imagen, preservar la memoria a través de representaciones visuales sugerentes y representarse como objeto de sus más profundos deseos. De esta manera, siglos después, las pantallas son producto de un proceso evolutivo que satisface tales necesidades: la televisión y su cosmovisión del mundo contenida en una pequeña caja, los videojuegos como escaparate mágico de la realidad y la computadora a modo de puente entre interlocutores de todas latitudes confirman que, regularmente, el darwinismo tecnológico no es únicamente de formas, sino también lo es de fondo, conlleva a las interacciones entre usuarios y, por lo tanto, a la indagación de su procesos comunicativos.

Y es que la tecnología genera cambios epistemológicos y ontológicos en la vida de los sujetos, modifica su concepción del mundo. Atendemos a la convergencia mediática y a la transformación de los escenarios comunicativos de la cotidianidad mediada por dispositivos altamente inteligentes, lo que Perez-Escoda, CastroZubizarreta y Fandos-Igado (2016) llaman "rasgos distintivos de la sociedad actual". Y es que, los dispositivos conectados a Internet inciden en cómo las nuevas y previas generaciones acceden al conocimiento, al entretenimiento e incluso, a sus propias relaciones inter personales. Según García-Galera, del HoyoHurtado y Fernández-Muñoz (2014), los jóvenes viven sumergidos en las redes sociales, por lo que las TIC se posicionan como una herramienta para informar, participar, debatir y movilizarse en los planos online y offline.

Sin embargo, la relación sistema de comunicación mediada-audiencia, hablando en términos de Luhmann (2000) o mediación desde Barbero (1998), resulta compleja cuando se institucionaliza. Esto es, a los jóvenes les cuesta creer en las instituciones y en los contenidos asociados a lo gubernamental; casos a nivel global son historias de desencanto respecto a la dieta mediática en el sector juvenil. Fenómeno no enteramente atribuible a los medios digitales, puesto que hay que recordar que en los años 70 tuvieron lugar en todo el mundo manifestaciones contra el legado de la modernidad, proceso conceptualizado como posmodernidad y que representó el cuestionamiento de los grandes relatos, el derrumbe de antiguos 
paradigmas (incluida la ciencia) y la "bienvenida" a la pluralidad de ideas (Lyotard, 1984). Por lo que acotar la actual crisis de confianza hacia las instituciones por parte de las audiencias desde la llegada del Internet sería reduccionista, en todo caso es el resultado del devenir histórico de las últimas décadas.

No obstante, Internet ha acentuado lo evidente al abrir la comunicación mediada a múltiples interlocutores e incidir en la producción, reproducción y consumo de mensajes. Dicho proceso, está provocando lo que Gros y Contreras (2006) denominan "competencias ciudadanas" para el desempeño activo en la sociedad democrática al suponer que, si los sujetos se desenvuelven frecuentemente en los espectros digitales, adquieren saberes para ser competentes en el uso de artefactos tecnológicos para la vida en democracia.

Tal condición de activismo, es una premisa recurrente en los nuevos modelos comunicativos y es que, si la realidad global se conoce a través de los medios, la sociedad debe entenderse como audiencia (Luhmann, 2000), pues prácticamente todo está en reflectores y pantallas digitales; asistimos al apremio de lo cotidiano. Una fotografía en el café de moda, actualizar el estado en redes sociales, Twittear, compartir un meme o video; son acciones comunes en la ecología mediática reciente.

Si bien, hay ejemplos importantes sobre activismo online/offline desde medios digitales: YoSoy132 (México), 15-M o movimiento de los indignados (España), Primavera Árabe (en diversos países de Medio Oriente) o el movimiento social por la educación en Chile, por citar algunos, existe pasividad en ciertos aspectos de la participación sociopolítica. De acuerdo a la encuesta de valores del IMJUVE (2013), a los jóvenes mexicanos de hasta 29 años no les gusta involucrarse en los destinos públicos, condición que refleja desencanto en las nuevas generaciones hacia la política.

Hoy en día son más visibles las inconformidades de los oprimidos, es lo que Cochrane, Antonczak y Wagner (2013) denominan como lo disruptivo de la era Post-Web 2.0, refiriéndose a que, si bien la tecnología mediada por el Internet ha abierto los procesos comunicativos hacia la participación de las audiencias, también ha permitido visibilizar los reclamos sociales.

Para O'reilly (2005) la era Web-2.0 es el viraje de Internet hacia la participación de los interlocutores, la Web es una plataforma que transita a modelos comunicativos centrados en el usuario final: contenidos creados por las audiencias, colaboración en línea, transmedia, entre otros. Web-2.0 es una locución utilizada por primera vez a mediados del 2000, y que algunos consideran superada por la de Web-3.0 (Wainewright, citado en $\mathrm{Hu}$ 2012) que dibuja la ubicuidad de la red, el almacenamiento en nube y el uso de metadatos. No obstante, más allá de definiciones precisas sobre Web 2.0 o 3.0, que no las hay, conviene aclarar que la literatura académica coincide respecto a la alta actividad de los usuarios en la ecología mediática digital, sobre todo cuando se trata de entretenimiento, pero conservadora en política. El fenómeno ha de situarse en la curva natural de lo novedoso, más cuando refleja el desencanto de lo que se observa desde las TIC y/o medios de comunicación, tal como los asuntos públicos, entonces es el momento de la era Post-Web, el tiempo donde convergen conceptualizaciones y herramientas tecnológicas en medio de la falta de consenso de los actores sociales sobre lo válido y lo obtuso del conocimiento. 
«Entre los jóvenes, las utopías revolucionarias de los setenta, el enojo y la frustración de los ochenta, han mutado, de cara al siglo veintiuno, hacia formas de convivencia que, pese a su acusado individualismo, parecen fundamentarse en un principio ético-político generoso: el reconocimiento explícito de no ser portadores de ninguna verdad absoluta en nombre de la cual ejercer un poder excluyente» (Regillo-Cruz, 2003:14).

Dicha falta de verdades no puede determinarse como algo negativo en la conformación cultural de las audiencias, es una condición latente ante las dificultades, bondades y condiciones del mundo en el que toca vivir. En ello, el valor de estar al corriente de las prácticas mediadas, usos y formas de obtención de significados en tiempos de la era Post-Web es apremiante. El paisaje comunicativo de la participación política ya no puede entenderse únicamente desde la movilización social tradicional o el acudir a las calles para emitir un voto, las estructuras simbólicas de los escenarios comunicativos recientes son expandidos a la cultura digital, sobre todo por los más jóvenes y deben estudiarse desde tales dimensiones.

\section{3. ¿Nativos \& inmigrantes digitales?}

Se ha advertido la complejidad de efectuar clasificaciones temporales o geográficas de los fenómenos sociales, por ejemplo, hablar de en qué era nos encontramos (mediática o tecnológicamente hablando). Con todo, ubicar la problematización del contexto desde la crisis de legitimidad social que viven las instituciones, y que conviene llamar era Post-Web, remite al referencial significativo de establecer categorías ejecutables para la exploración empírica de los sujetos estudiados y posibilitar así, supuestos hipotéticos para el contexto analizado.

Prensky (2001) a inicios del nuevo milenio, acuñó los términos nativos e inmigrantes digitales. Los primeros nacidos con las TIC, se suponían con mayores destrezas informáticas, mientras que los segundos tendrían que adaptarse a ellas para atender las exigencias del mundo digital. "Esta divisoria entre migrantes y nativos tiene filiaciones importantes pero es de una fabulosa actualidad que, antes que temer, deberíamos deconstruir y eventualmente rediseñar" (Piscitelli, 2006: 181).

Es curioso que al examinar los términos nativos e inmigrantes digitales, dentro de las bases académicas de datos, se encuentran debates importantes en el tema. Están quienes consideran que hacer dicha clasificación podría resultar hasta discriminatorio, otros como: Piscitelli (2006; 2008), Caldeiro-Pedreira \& AguadedGómez (2015), Grijalva-Verdugo (2016), Córdoba Mercado \& Pérez Córdoba (2016), Sanchez-Espinoza \& Castro-Ricalde (2013), Leymonié (2015) y muchos más, argumentan que hay rasgos distintivos asociados a la edad y al acceso tecnológico que ratifican las brechas entre nativos e inmigrantes digitales aunque no siempre en sentido positivo, en otras palabras, no es una condición constante que los más jóvenes posean altas competencias mediáticas o saberes digitales, más bien existen factores complementarios a la luz de la tecnología. 
Esta aclaración es necesaria debido a que, en la investigación se opta por agrupar a los sujetos en nativos e inmigrantes digitales; el objetivo fue explorar las posibles diferencias entre jóvenes $y$ adultos en torno a la participación sociopolítica. Para ello, se usa la siguiente delimitación teórica-temporal:

«Los nativos digitales, son aquellos sujetos menores de 30 años que han crecido con la tecnología y, por lo tanto, tienen una habilidad innata en el lenguaje y en el entorno digital. Las herramientas tecnológicas ocupan un lugar central en sus vidas y dependen de ellas para todo tipo de cuestiones cotidianas como estudiar, relacionarse, comprar, informarse o divertirse») (García, Portillo, Romo, \& Benito, 2007:3 )

En tal, es viable operacionalizar el hipótetico de que al estar los más jóvenes especialmente habituados a los medios digitales y poseer mayor cultura digital (ideal), en consecuencia estarían más adelantados respecto a la participación sociopolítica. Sin embargo, para evitar la dicotomía de la que se hablaba anteriormente, se generó una nueva clasificación a fin de identificar su comportamiento en las pruebas estadísticas del estudio, la variable se denomina uso de medios digitales; resulta de sumar las horas que los sujetos dedican a redes sociales: Facebook, YouTube, Instagram, WhatsApp, entre otras, y no necesariamente estar dentro del rango menores de 30 años (nativos digitales) o por encima de este (inmigrantes digitales). En resumen, son las variables grupo etario (nativos e inmigrantes digitales) y uso de medios digitales las que permiten contrastar cada uno de los ítems de la presente investigación.

\section{Método e instrumentos}

La metodología consta de tres etapas. En la primera, se construyen las categorías analíticas desde la literatura existente, lo que da paso a la elaboración de un cuestionario tipo escala Likert a cinco puntos; en él, los sujetos se enfrentan ante el acuerdo y desacuerdo de las premisas o ítems propuestos, se compone por 58 afirmaciones, en este artículo se examinan ocho referidas al constructo participación sociopolítica desde medios digitales, su selección demostró un valor aceptable de consistencia interna (alfa de Cronbach de 0,720). El instrumento se aplicó de julio a noviembre de 2016 de forma online, cara a cara, impreso y telefónicamente.

La muestra resulta en 386 sujetos del estado de Sinaloa elegidos aleatoriamente en las principales ciudades de la entidad, se procedió conforme a los criterios del Instituto Nacional Electoral (INE). El arranque fue aleatorio por cuotas de sexo, edad y escolaridad; el cálculo se adquiere de un padrón electoral de 2,064,508 sujetos (Instituto Electoral del Estado de Sinaloa, 2016) alcanzando un intervalo de confianza del $95 \%$. 


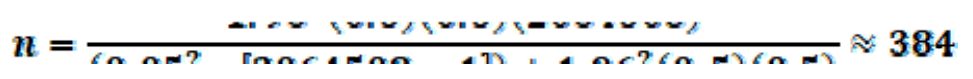

En la aplicación del cuestionario el valor de " $n$ " fue 386, para tener un margen por encima de lo calculado con la formula $(n=384)$. La última etapa de la investigación, consistió en agrupar a los sujetos a partir de las variables de grupo etario (nativos e inmigrantes digitales) y uso de medios (uso bajo, medio y alto) para proceder con los análisis e interpretación de datos y presentarlos en distintas publicaciones. Justamente, el alcance de la investigación se sitúa en los estudios descriptivos, exploratorios y de carácter relacional; se ejecutaron las pruebas no paramétricas $U$ de Mann-Whitney, Chi-cuadrado y $H$ de Kruskal-Wallis, también se obtuvieron frecuencias, porcentajes y comparación de medidas de tendencia central para responder a las interrogantes planteadas en el artículo. Los datos fueron procesados en el programa estadístico SPSS versión 21.

\section{Resultados}

Conseguidas las frecuencias de uso de medios digitales por separado: prensa digital, Facebook, WhatsApp, Instagram, YouTube y otras redes sociales, se construyó una nueva variable sumando las anteriores; un índice que permitió reconocer la utilización de los medios a partir de las respuestas de los sujetos de la muestra, y que se denominó uso de medios digitales. Los datos son organizados en relación a la distribución en los cuartiles $(\mathrm{C} 1, \mathrm{C} 2, \mathrm{C} 3$ y $\mathrm{C} 4)$; de ahí se categorizan en uso bajo, medio y alto, es decir, en la primera etiqueta (uso bajo) se agrupan las personas que utilizan poco la tecnología o están en el mínimo de $\mathrm{C} 1$; uso medio, quienes se ubican en el máximo del $\mathrm{C} 1$ hasta el mínimo de $\mathrm{C} 3$, y uso alto; compuesto por los ciudadanos que pasan más horas en plataformas digitales o están en el máximo de $\mathrm{C} 3$ y $\mathrm{C} 4$. Se obtiene $24,3 \%$ en uso bajo, $47,7 \%$ uso medio y $28,0 \%$ uso alto $(\mathrm{N}=386)$.

¿Pero quiénes son los sujetos que integran cada una de las etiquetas obtenidas? Para ello, la escolaridad podría ofrecer un paisaje descriptivo de las audiencias analizadas, tal como se muestra en la tabla 1. 
Tabla 1. Uso de medios en relación a la escolaridad

\begin{tabular}{|c|c|c|c|}
\hline $\begin{array}{c}\text { Variable de } \\
\text { agrupación }\end{array}$ & $\begin{array}{c}\text { Uso bajo } \\
(\%)\end{array}$ & $\begin{array}{c}\text { Uso medio } \\
(\%)\end{array}$ & $\begin{array}{c}\text { Uso alto } \\
(\%)\end{array}$ \\
\hline General & 24,3 & 47,7 & 28,0 \\
\hline Escolaridad & & & \\
\hline Sin estudios & 100,0 & 0,0 & 0,0 \\
\hline Primaria & 81,3 & 18,7 & 0,0 \\
\hline Secundaria & 44,0 & 40,0 & 16,0 \\
\hline Bachillerato & 18,2 & 50,0 & 31,8 \\
\hline Licenciatura & 20,1 & 50,0 & 29,9 \\
\hline Posgrado & 14,3 & 57,1 & 28,6 \\
\hline
\end{tabular}

Fuente: Elaboración propia/ trabajo de campo

Se distingue que la variable de agrupación escolaridad marca una correspondencia con el uso de medios digitales en la entidad, ya que los sujetos sin estudios usan poco las TIC (100,0\% uso bajo), mientras que el consumo más alto se detecta en los individuos con bachillerato $(31,8 \%)$, ligeramente superior a los sujetos con licenciatura $29,9 \%$. El hallazgo corresponde con el fenómeno de la brecha digital en los países del tercer sector, tal como argumenta Tello-Leal (2007). La situación, coincide al congregar a los individuos por grupo etario (nativos e inmigrantes digitales). Ver tabla 2.

Tabla 2. Uso de medios por grupo etario

\begin{tabular}{|c|c|c|c|}
\hline $\begin{array}{c}\text { Variable de } \\
\text { agrupación }\end{array}$ & $\begin{array}{c}\text { Uso bajo } \\
(\%)\end{array}$ & $\begin{array}{c}\text { Uso medio } \\
(\%)\end{array}$ & $\begin{array}{c}\text { Uso alto } \\
(\%)\end{array}$ \\
\hline Grupo etario & & & \\
\hline Nativos digitales & 14,8 & 49,3 & 35,9 \\
\hline $\begin{array}{c}\text { Inmigrantes } \\
\text { digitales }\end{array}$ & 46,6 & 44,0 & 9,4 \\
\hline
\end{tabular}

Fuente: Elaboración propia/ trabajo de campo

Se encuentra que la Media estadística de la variable uso de medios digitales de nativos es de 21,28 (DT=5,379) y la de inmigrantes de 15,65 (DT=6,497), siendo consistentes con la variable escolaridad. La tabla 2 , da cuenta de que en términos porcentuales los nativos digitales pasan más tiempo en medios virtuales. De los inmigrantes apenas el 9,4\% posee uso alto, frente al 35,9\% de nativos, por lo que el hipotético de que los más jóvenes usan más la tecnología es sólido, tal como arroja la prueba no paramétrica de Chi-Cuadrada $\left(\mathrm{X}^{2}(42, \mathrm{~N}=386)=54,315 ; \mathrm{p}=0,000\right)$. 
Lo antepuesto, corrobora la pertinencia de la clasificación nativos e inmigrantes digitales y uso de medios digitales como variables de agrupación al percibirse diferencias intergrupos; hecho esto, se buscó conocer las diferencias entre las puntuaciones medias de estos. Al no cumplirse con la homocedasticidad de la muestra en ninguno de los ocho ítems, se procede con la prueba $U$ de MannWhitney cuyos resultados se muestran en la tabla 3.

Tabla 3. Diferencia de medias por nativos e inmigrantes digitales. Prueba $U$ de Mann-Whitney

\begin{tabular}{|c|c|c|c|c|c|c|c|c|c|}
\hline \multirow[t]{2}{*}{ Ítem } & \multicolumn{3}{|c|}{$\begin{array}{l}\text { Nativos } \\
\text { digitales }\end{array}$} & \multicolumn{3}{|c|}{$\begin{array}{l}\text { Inmigrantes } \\
\text { digitales }\end{array}$} & \multirow{2}{*}{$\begin{array}{l}\text { U de } \\
\text { Mann- } \\
\text { Whitney }\end{array}$} & \multirow[t]{2}{*}{ Z } & \multirow[t]{2}{*}{ Sig. } \\
\hline & $\mathrm{N}$ & $\begin{array}{l}\text { Medi } \\
\mathrm{a}\end{array}$ & DT & $\mathrm{N}$ & $\begin{array}{l}\text { Medi } \\
\text { a }\end{array}$ & DT & & & \\
\hline $\begin{array}{l}\text { Difundo, } \\
\text { produzco o } \\
\text { comento } \\
\text { memes, dado } \\
\text { que me } \\
\text { ayudan a } \\
\text { generar } \\
\text { conciencia } \\
\text { sobre la } \\
\text { política }\end{array}$ & 26 & 3,20 & $\begin{array}{l}1,41 \\
1\end{array}$ & $\begin{array}{l}11 \\
4\end{array}$ & 3,28 & $\begin{array}{l}1,63 \\
8\end{array}$ & 14834,00 & $\begin{array}{l}- \\
0,51 \\
8\end{array}$ & $\begin{array}{l}0,60 \\
5\end{array}$ \\
\hline $\begin{array}{l}\text { Creo que si } \\
\text { participo en } \\
\text { política } \\
\text { habrá } \\
\text { mayores } \\
\text { posibilidades } \\
\text { de mejorar la } \\
\text { calidad de } \\
\text { vida del país }\end{array}$ & 26 & 3,28 & $\begin{array}{l}1,39 \\
5\end{array}$ & $\begin{array}{l}10 \\
6\end{array}$ & 3,43 & $\begin{array}{l}1,56 \\
9\end{array}$ & 13435,50 & $\begin{array}{l}- \\
1,21 \\
4\end{array}$ & $\begin{array}{l}0,22 \\
4\end{array}$ \\
\hline $\begin{array}{l}\text { Comento en } \\
\text { redes } \\
\text { sociales de } \\
\text { medios } \\
\text { informativos } \\
\text { (Noroeste, El } \\
\text { Universal, } \\
\text { entre otros) } \\
\text { la } \\
\text { información } \\
\text { que publican }\end{array}$ & 27 & 4,04 & $\begin{array}{l}1,20 \\
9\end{array}$ & $\begin{array}{l}11 \\
3\end{array}$ & 3,76 & $\begin{array}{l}1,48 \\
4\end{array}$ & 14013,50 & $\begin{array}{l}- \\
1,37 \\
1\end{array}$ & $\begin{array}{l}0,17 \\
0\end{array}$ \\
\hline
\end{tabular}




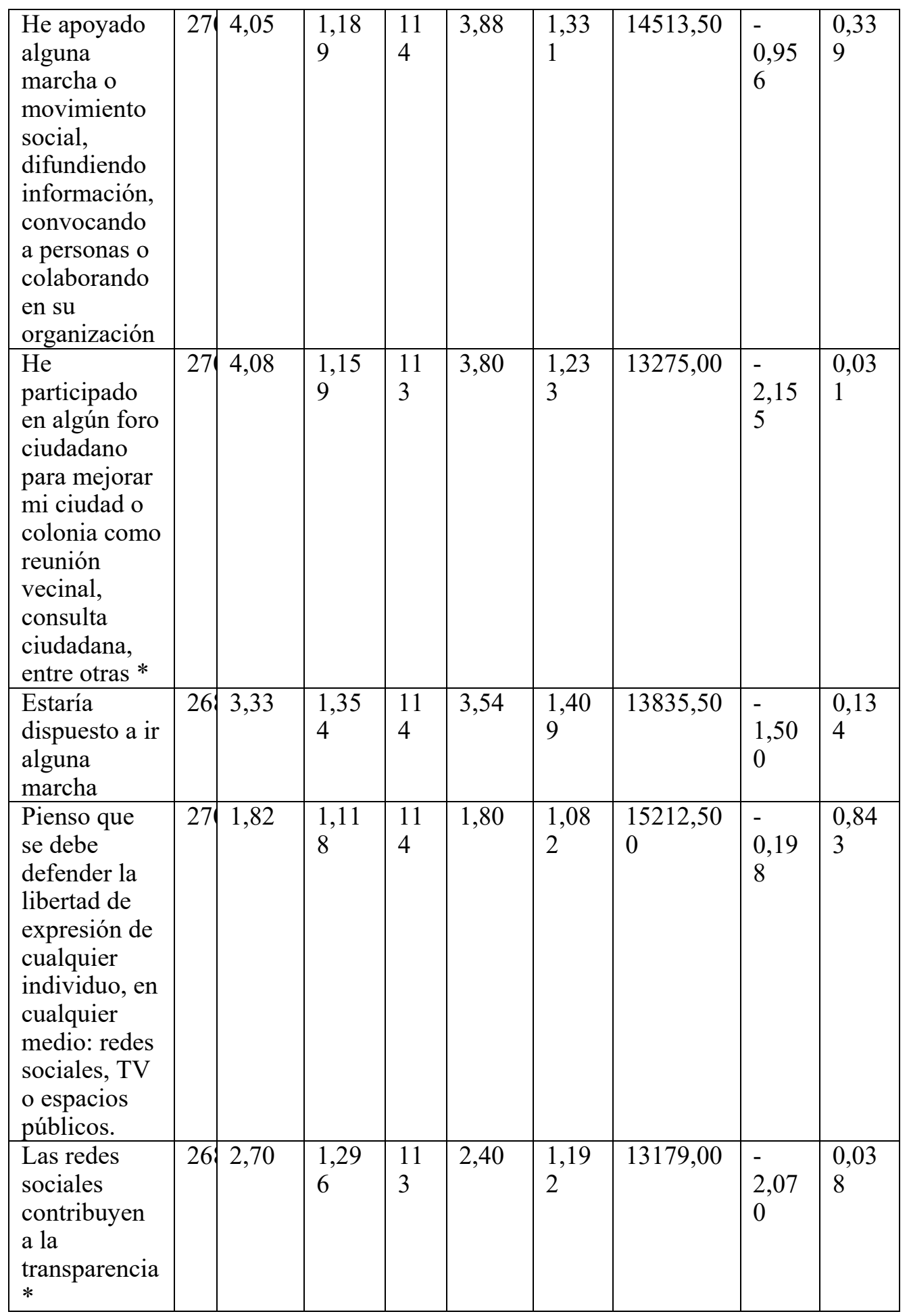


* Diferencia estadísticamente significativa $\mathrm{p}<0,05$

Fuente Elaboración propia/ trabajo de campo.

Únicamente en los ítems "he participado en algún foro ciudadano para mejorar mi ciudad o colonia como reunión vecinal, consulta ciudadana, entre otras" ( $\mathrm{Z}=$ $2,155, \mathrm{p}<0,05)$ y "las redes sociales contribuyen a la transparencia" presentan diferencias estadísticamente significativas $(Z=-2,070, p<0,05)$. Llama la atención que la Media de las preguntas 7 y 8 en relación con la libertad de expresión y el papel de las redes sociales en asuntos de transparencia son mal evaluados por ambos subgrupos. En una escala a cinco puntos, donde uno es el valor más bajo y cinco el más alto, obtener una puntuación menor a dos (Media) para el primer ítem, y menor a tres para el segundo, denota descontento social sobre la política, tal como advierte Regillo-Cruz (2003).

Para explorar las respuestas en función de cómo los individuos emplean los medios digitales (uso bajo, medio y alto), se recurrió a la prueba de $H$ de KruskalWallis al ser más de dos grupos independientes. El análisis estadístico equivale a la ANOVA de un factor y compara los rangos entre la variable de agrupación en proporción al consumo de medios digitales; lo ítems refieren a participar en redes sociales comentando contenido o información política, apoyar marchas y/o manifestaciones, producir contenidos políticos como memes, entre otros aspectos.

Tabla 4. H de Kruskal-Wallis, diferencia por uso bajo, medio y alto

\begin{tabular}{|l|c|c|c|c|c|c|}
\hline \multicolumn{1}{|c|}{ Ítem } & \multicolumn{3}{|c|}{ Rango promedio } & H & GL & p \\
\hline $\begin{array}{l}\text { Uso } \\
\text { bajo }\end{array}$ & $\begin{array}{c}\text { Uso } \\
\text { medio }\end{array}$ & $\begin{array}{c}\text { Uso } \\
\text { alto }\end{array}$ & & & \\
\hline $\begin{array}{l}\text { Difundo, produzco o } \\
\text { que me ayudan a } \\
\text { generar conciencia } \\
\text { sobre la política }\end{array}$ & 226,53 & 190,04 & 165,90 & 15,862 & 2 & 0,00 \\
\hline $\begin{array}{l}\text { Creo que si participo } \\
\text { en política habrá } \\
\text { mayores posibilidades } \\
\text { de mejorar la calidad } \\
\text { de vida del país }\end{array}$ & 171,26 & 187,93 & 196,99 & 2,978 & 2 & 0,22 \\
\hline $\begin{array}{l}\text { Comento en redes } \\
\text { sociales de medios } \\
\text { informativos } \\
\text { (Noroeste, El }\end{array}$ & 222,70 & 186,80 & 174,33 & 12,270 & 2 & 0,02 \\
$\begin{array}{l}\text { Universal, entre otros) } \\
\text { la información que } \\
\text { publican. }\end{array}$ & & & & & & \\
\hline He apoyado alguna & 213,48 & 200,72 & 160,50 & 15,617 & 2 & 0,00 \\
\hline
\end{tabular}




\begin{tabular}{|l|l|l|l|l|l|l|}
\hline $\begin{array}{l}\text { marcha o movimiento } \\
\text { social, difundiendo } \\
\text { información, } \\
\text { convocando a } \\
\text { personas o } \\
\text { colaborando en su } \\
\text { organización. }\end{array}$ & & & & & & \\
\hline $\begin{array}{l}\text { He participado en } \\
\text { algún foro ciudadano } \\
\text { para mejorar mi } \\
\text { ciudad o colonia como } \\
\text { reunión vecinal, } \\
\text { consulta ciudadana, } \\
\text { entre otras }\end{array}$ & 226,15 & 182,30 & 179,35 & 13,383 & 2 & 0,01 \\
\hline $\begin{array}{l}\text { Estaría dispuesto a ir } \\
\text { alguna marcha }\end{array}$ & 226,88 & 192,07 & 159,49 & 19,511 & 2 & 0,00 \\
\hline $\begin{array}{l}\text { Pienso que se debe } \\
\text { defender la libertad de } \\
\text { expresión de cualquier } \\
\text { individuo, en } \\
\text { cualquier medio: redes } \\
\text { sociales, TV o } \\
\text { espacios públicos. }\end{array}$ & 216,27 & 181,83 & 190,12 & 7,371 & 2 & 0,02 \\
\hline $\begin{array}{l}\text { Las redes sociales } \\
\text { contribuyen a la } \\
\text { transparencia }\end{array}$ & 217,68 & 187,80 & 173,92 & 8,673 & 2 & 0,01 \\
\hline
\end{tabular}

Fuente: Elaboración propia/trabajo de campo

La prueba Kruskal-Wallis demuestra diferencias por grupos (uso bajo, medio y alto) en todos los ítems y diferencias estadísticas en 7 , excepto en "creo que si participo en la política habrá mayores posibilidades de mejorar la calidad de vida del país" ( $\mathrm{p}=0,226)$, lo que comprueba el hipotético de que la cultura de la participación sociopolítica se conecta a cómo los sujetos emplean las TIC ¿pero es esto necesariamente positivo? Es decir, ¿quiénes mantienen uso tecnológico alto participan más que los de uso bajo? Para responder, se muestran los ítems con las Medias en cada subgrupo, donde uno significa poca identificación con la premisa y cinco mucha identificación con lo planteado en el ítem. 
Tabla 5. Comparación de Media estadística por grupos bajo, medio y alto

\begin{tabular}{|l|l|l|l|l|l|l|l|l|l|}
\hline & \multicolumn{3}{|c|}{ Uso bajo } & \multicolumn{3}{c|}{ Uso medio } & \multicolumn{3}{c|}{ Uso alto } \\
\hline \multicolumn{1}{|c|}{ Item } & N & Media & Moda & N & Media & Moda & N & Media & Moda \\
\hline $\begin{array}{l}\text { Difundo, } \\
\text { produzco o } \\
\text { comento } \\
\text { memes, dado } \\
\text { que me } \\
\text { ayudan a } \\
\text { generar } \\
\text { conciencia } \\
\text { sobre la } \\
\text { política }\end{array}$ & 92 & 3,70 & 5 & 183 & 3,20 & 5 & 108 & 2,87 & 2 \\
\hline $\begin{array}{l}\text { Creo que si } \\
\text { participo en } \\
\text { política habrá } \\
\text { mayores } \\
\text { posibilidades } \\
\text { de mejorar la } \\
\text { calidad de } \\
\text { vida del país }\end{array}$ & 89 & 3,09 & 1 & 178 & 3,36 & 5 & 105 & 3,46 & 5 \\
\hline $\begin{array}{l}\text { Comento en } \\
\text { redes sociales } \\
\text { de medios } \\
\text { informativos } \\
\text { (Noroeste, El } \\
\begin{array}{l}\text { Universal, } \\
\text { entre otros) la } \\
\text { información } \\
\text { que publican }\end{array} \\
\begin{array}{l}\text { He apoyado } \\
\text { alguna marcha } \\
\text { o movimiento } \\
\text { social, } \\
\text { difundiendo } \\
\text { información, } \\
\text { convocando a } \\
\text { personas o } \\
\text { colaborando } \\
\text { en su } \\
\text { organización }\end{array}\end{array}$ & 93 & 4,32 & 5 & 182 & 3,92 & 5 & 108 & 3,72 & 5 \\
\hline
\end{tabular}




\begin{tabular}{|c|c|c|c|c|c|c|c|c|c|}
\hline $\begin{array}{l}\text { He participado } \\
\text { en algún foro } \\
\text { ciudadano } \\
\text { para mejorar } \\
\text { mi ciudad o } \\
\text { colonia como } \\
\text { reunión } \\
\text { vecinal, } \\
\text { consulta } \\
\text { ciudadana, } \\
\text { entre otras }\end{array}$ & 92 & 4,39 & 5 & 183 & 3,90 & 5 & 108 & 3,81 & 5 \\
\hline $\begin{array}{l}\text { Estaría } \\
\text { dispuesto a ir } \\
\text { alguna marcha }\end{array}$ & 93 & 3,84 & 5 & 183 & 3,41 & 5 & 106 & 2,97 & 3 \\
\hline $\begin{array}{l}\text { Pienso que se } \\
\text { debe defender } \\
\text { la libertad de } \\
\text { expresión de } \\
\text { cualquier } \\
\text { individuo, en } \\
\text { cualquier } \\
\text { medio: redes } \\
\text { sociales, TV o } \\
\text { espacios } \\
\text { públicos. }\end{array}$ & 93 & 2,12 & 1 & 183 & 1,68 & 1 & 108 & 1,77 & 1 \\
\hline $\begin{array}{l}\text { Las redes } \\
\text { sociales } \\
\text { contribuyen a } \\
\text { la } \\
\text { transparencia }\end{array}$ & 91 & 2,95 & 3 & 182 & 2,56 & 3 & 108 & 2,41 & 1 \\
\hline
\end{tabular}

Fuente: Elaboración propia/trabajo de campo

Se descubren diferencias expresadas en las medidas de tendencia central (Media y Moda) entre grupos, contrario a lo que se pudiera pensar de quienes tienen más acercamiento tecnológico (uso alto), pues no son ellos quienes más participan. Por ejemplo, al consultarles sobre la producción de memes para generar conciencia política, quienes tienen la puntuación más baja son quienes pasan más horas en medios digitales (Media de 2,87), y quienes más comparten, difunden y/o producen contenidos son usuarios con menor uso (Media 3,70).

Concerniente a la confianza de participar en política, los más desencantados son los ciudadanos de uso bajo con una Media de 3,09 (y Moda estadística de 1) a diferencia de quienes poseen uso medio y alto (Medias de 3,36 y 3,46 respectivamente).

Otro elemento constituyente a la cultura digital y a la participación sociopolítica es la actividad generada por las nuevas audiencias en las ecologías mediáticas, 
premisa popularizada en la era Post-Web, y que complementa el relato de la transparencia y la capacidad de retroalimentación brindada por las novedosas plataformas digitales. En tal vertiente, se pregunta a los encuestados si comentan en redes sociales o participan en las páginas virtuales de los medios informativos noticiosos (prensa digital local y nacional) en contenidos políticos. La correlación es inversa, quienes usan menos las TIC comentan más las redes sociales de la prensa (Media 4,32), tal como Noroeste o El Universal ${ }^{3}$, puntuación que disminuye en los otros grupos (sujetos de uso medio $=3,92$ y sujetos de uso alto $=3,72$ ), ¿a qué se debe esta situación? El resultado arroja que utilizar la tecnología no es precisamente sinónimo de participación.

Lo anterior confirma el supuesto de que, si bien la participación de nativos e inmigrantes digitales es diversificada, no siempre es en función de involucrarse, puesto que los mayores de 30 años se identifican más con asistir a una marcha (Media 3,54) que los más jóvenes o nativos digitales (Media 3,33), tal como se muestra en la tabla 3. Circunstancia que coincide al agrupar a los sujetos desde la variable de contraste de usos mediáticos, en la que el subgrupo menos tecnológico (uso bajo) comparte la acción social como parte de su composición.

En ese mismo sentido, la intervención sociopolítica contemporánea no puede concebirse, únicamente, como tomar las calles o accionar mecanismos de protesta, se debe considerar la producción de mensajes comunicativos mediados con impactos en la otredad, ahí que el ítem "he apoyado alguna marcha o movimiento social difundiendo información, convocando a personas o colaborando en su organización", arroja que los más jóvenes son más interactivos (Media 4,05). Ver tabla 3. En tal, los nuevos escenarios sociopolíticos son complejos, y son más naturales a quienes han incorporado desde más jóvenes las TIC y los medios a sus vidas desde la edad temprana.

\section{Conclusiones}

Las variables de agrupación del estudio (usos mediáticos y grupo etario), permiten visualizar diferencias en la participación sociopolítica de las audiencias de la era Post-Web, donde si bien, los jóvenes exteriorizan mayor proximidad a la tecnología, no siempre despliegan dispositivos de participación en la red, llámese convocar a otros para movilizaciones o asistir a foros en su colonia.

Los inmigrantes digitales declaran apertura significativa al pensamiento sociopolítico al evaluar positivamente el instrumento de investigación en los ítems concernientes a la acción social. Tanto nativos como inmigrantes digitales desconfían de la contribución de los medios a la democracia, por ejemplo, al consultarles si consideran que las redes sociales ayudan a la transparencia los hallazgos son preocupantes, prevalece la negatividad en la ciudadanía. En ese sentido, es ineludible que se indaguen variables más cualitativas que recuperen dicha condición.

\footnotetext{
${ }^{3}$ Noroeste y El Universal son periódicos con gran impacto en la población. El primero de carácter local, y el segundo nacional e internacional. Ambos, son de los que más contenido generan para redes sociales.
} 
La ciudadanía mediática pese a manifestar descontento sobre política, posee usos digitales importantes, por lo que se tendría que reflexionar si la crisis de confianza sociopolítica que distinguen nativos e inmigrantes digitales, es la expresión de lo vivido en el mundo offline. En tal, el microcosmos dibujado en esta entidad mexicana (Sinaloa) da cuenta que la globalidad está presente en la localidad, la conectividad representa un punto de encuentro para significar los medios, la cultura y la participación individual y colectiva de quienes hacen parte de esta era hipercomunicada y cambiante donde urgen investigaciones potentes para caracterizar los procesos comunicativos mediados.

\section{Referencias bibliográficas}

Anderson, C., Regan, P., \& Ostergard, R. (2002). "Political Repression and Public Perceptions of Human Rights". Political Research Quarterly, 55, 2, 439-456. Doiorg.proxy3.library.mcgill.ca/10.1177/106591290205500208. Consultado 1 de mayo de 2017.

Caldeiro-Pedreira, M.C., Aguaded Gómez, J.I.: "Estoy aprendiendo, no me molestes, la competencia mediática como forma de expresión crítica de nativos e inmigrantes digitales". Redes. Revista Hispana para el Análisis de Redes Sociales. № 12, págs. $27-$ 45, (2015). ISSN: 1579-0185 Disponible en http://rabida.uhu.es/dspace/bitstream/handle/10272/12275/Estoy_aprendiendo.pdf?sequ ence $=2$. Consultado el 3 de febrero de 2017.

Gros Salvat, B., \& Contreras, D. (2006). "La alfabetización digital y el desarrollo de competencias ciudadanas". Revista Iberoamericana de Educación (OEI), 2006, núm. 42, $\quad$ p. 103-125. Disponible en http://diposit.ub.edu/dspace/bitstream/2445/57176/1/567513.pdf. Consultado el 1 de julio de 2017.

Martín-Barbero, J. (1998). De los medios a las mediaciones: comunicación, cultura y hegemonía. Andrés Bello: Convenio.

Foucault, M.; Varela, J.; ÁlvareZ-Urías, F. La voluntad de saber. Madrid: Siglo XXI editores.

García-Galera, M.; Del Hoyo Hurtado, M.; Fernández-Muñoz, C. (2017). "Las redes sociales en la cultura digital: percepción, participación, movilización. Revista de la Asociación Española de Investigación de la Comunicación, v. 1, n. 1, p. 12-18, mayo 2014. ISSN 2341-2690. Disponible en: http://www.revistaeic.eu/index.php/raeic/article/view/19. Consultado el 13 septiembre de 2017.

Leymonié, J. (2015). "Nativos e inmigrantes digitales: ¿cómo aprendemos y enseñamos?". Dixit, no $12, \quad$ p. 10-19. Disponible en https://www.ucu.edu.uy/index.php/revistadixit/article/view/292/273. Consultado el 2 de mayo de 2017.

Caldeiro-Pedreira, M., \& Aguaded-Gómez, J. (2015). Estoy aprendiendo, no me molestes. La competencia mediática como forma de expresión crítica de nativos e inmigrantes digitales. Redes. Revista hispana para el análisis de redes sociales (12), 27-45. 
Lipovetsky, G., \& Serroy, J. (2009). La pantalla global. Cultura mediática y cine en la era hipermoderna. Barcelona: Anagrama.

Loreto, M., Silva, C., \& Hernández, A. (2010). ¿En que ciudadanía creen los jóvenes? Creencias, aspiraciones de ciudadanía y motivaciones para la participación sociopolítica. PsyKhe, 19(2), 25-37.

Córdoba Mercado, M., \& Pérez Córdoba, A. (2016). Análisis de las estrategias de aprendizaje empleadas por nativos e inmigrantes digitales en un ambiente virtual. Simpósio Internacional de Educaçao a Distància (págs. 1-13). EnPed.

Grijalva-Verdugo, A. (2016). "Competencia mediática en nativos digitales: evaluación desde la educación básica”. En T. Carrillo-Montoya, A. Grijalva-Verdugo, M. UrreaZazueta (Ed.), Espacios en transición: educación y desarrollo social Ciudad de México: Ediciones del Lirio, Universidad de Occidente y UPES, pp.31-48.

Grijalva-Verdugo, A., \& Mondaca-Cota, A. (2017). “ ¡Ni participo, ni me gusta! La ciudadanía mediática ante los movimientos sociales en México: estudio desde lo local". En A. Cabral, C. Bolaño , D. Araujo, F. Andacht, \& F. Paulino, Nuevos conceptos y territorios en América Latina. Sāo José dos Pinhais: Página 42, pp. 440-462.

Grijalva-Verdugo, A., \& Moreno-Candil, D. (2017). "Empoderamiento social en contextos violentos mexicanos mediante la competencia mediática" [Social Empowerment in Mexican Contexts through Media Competence]. Comunicar(53). https://doi.org/10.3916/C53-2017-03

INJUVE. (2013). Encuesta Nacional de Valores en Juventud 2012. Instituto Mexicano de la Juventud . CDMX: IMJUVE, Gobierno Federal, Secretaria de Educación Pública.

Instituto Electoral del Estado de Sinaloa. (2016). IEES Instituto Electoral del Estado de Sinaloa . Recuperado el 31 de Enero de 2017, de IEES Instituto Electoral del Estado de Sinaloa : http://www.cee-sinaloa.org.mx/publico/principal/index.aspx

Perez-Escoda, A., Castro-Zubizarreta, A., \& Fandos-Igado, M. (2016). "La competencia digital de la Generación Z: claves para su introducción curricular”. Comunicar, 71-80. Doi http://dx.doi.org/10.3916/C49-2016-07

Piscitelli , A. (2008). "Nativos e inmigrantes digitales: una dialéctica intrincada pero indispensable”. En R. Carneiro, J. Toscano, \& T. Díaz, Los desafíos de las TIC para el cambio educativo (págs. 71-78). Madrid: Fundación Santillana.

Piscitelli, A. (2006). "Nativos e inmigrantes digitales: ¿brecha generacional, brecha cognitiva, o las dos juntas y más aún?” Revista Mexicana de Investigación Educativa, 11(28), 11-28. Disponible en http://www.redalyc.org/articulo.oa?id=14002809. Consultado el 3 de mayo de 2017.

Prensky, M. (2001). Digital Natives, Digital Immigrants. New York: MCB University Press.

Regillo-Cruz, R. (2003). Emergencia de las culturas juveniles estrategias del desencanto . Bogota : Grupo Editorial Norma.

Ruelas, A. (2016). "Redes sociales digitales y participación. El movimiento No al alza del transporte urbano en Culiacán". Razón y Palabra , 279-299. Disponible en http://www.redalyc.org/articulo.oa?id=199545660018. Consultado el 1 de septiembre de 2017. 
Sanchez-Espinoza, A., \& Castro-Ricalde, D. (2013). "Cerrando la brecha entre nativos e inmigrantes digitales a través de las competencias informáticas e informacionales". Apertura. Revista de innovación educativa, 5(2), 6-15. Disponible en http://www.udgvirtual.udg.mx/apertura/index.php/apertura/article/view/413. Consultado el 3 de agosto de 2017.

Tello-Leal, E. (2007). "Las tecnologías de la información y comunicaciones (TIC) y la brecha digital: su impacto en la sociedad". Revista de Universidad y Sociedad del Conocimiento, 4(2), 1-8. Disponible en http://www.uoc.edu/rusc/4/2/dt/esp/tello.pdf. Consultado el 3 de febrero de 2017.

\section{Agradecimientos}

A los jóvenes de verano científico: Mariana Tizoc, Daniela Covarrubias e Iván Valenzuela, alumnos de Comunicación de la Universidad de Occidente, así como a Iris Cebreros, maestrante de Gobierno y Políticas Públicas de la misma casa de estudios. Gracias por su invaluable apoyo para la recolección de los datos de esta investigación. 